\title{
Anti-inflammatory functions of Houttuynia cordata Thunb. and its compounds: A perspective on its potential role in rheumatoid arthritis (Review)
}

\author{
$\mathrm{JUN} \mathrm{LI}^{1,2}$ and FUTAO ZHAO ${ }^{1}$ \\ ${ }^{1}$ Department of Rheumatology and Immunology, Shanghai Third People's Hospital, School of Medicine, \\ Shanghai Jiao Tong University, Shanghai 201999; ${ }^{2}$ Department of Internal Medicine, \\ Bengbu Medical College, Bengbu, Anhui 233030, P.R. China
}

Received August 12, 2014; Accepted March 25, 2015

DOI: 10.3892/etm.2015.2467

\begin{abstract}
The aim of this review was to take a look at the anti-inflammatory functions of Houttuynia cordata Thunb. (HCT) that have been illustrated in the literature and to explore new fields in which HCT could be used in the future. The use of HCT has been described in broad inflammatory domains, where it has exhibited a variety of activities, including antiviral, antibacterial, antiparasitic and immunostimulant activity, with high efficiency, mild features and definite therapeutic effects. The numerous anti-inflammatory functions of HCT have demonstrated that HCT has wide application prospects. New uses of HCT and the full extent of its utilization await further investigation. The basic pathological change of rheumatoid arthritis (RA) is synovial proliferation which leads to joint destruction in the long-term. There are types of drugs that have been used clinically for patients with RA, however, due to their side-effects or high prices their broad usage is limited. A safe and low-cost drug is urgently required to be developed for the clinical usage of patients with RA. Thus, HCT has the potential to be a good candidate in the treatment of rheumatoid arthritis.
\end{abstract}

\section{Contents}

1. Introduction

2. Antiviral activity

3. Antibacterial activity

4. Antiparasitic activity

Correspondence to: Dr Futao Zhao, Department of Rheumatology and Immunology, Shanghai Third People's Hospital, School of Medicine, Shanghai Jiao Tong University, 280 Mohe Road, Shanghai 201999, P.R. China

E-mail: zhaofutao@aliyun.com

Key words: Houttuynia cordata Thunb., anti-inflammatory functions, rheumatoid arthritis
5. Other anti-inflammatory activity

6. Effects on immunity

7. Conclusion

\section{Introduction}

Houttuynia cordata Thunb. (HCT) is a perennial herbaceous plant that grows in the wild in moist and shady locations in Asian countries, including China, Japan and India. HCT has antiviral, antibacterial, immunostimulant and other anti-inflammatory effects (1), which have led it to being widely used in the pharmaceutical industry and folk medicine (2-5). In recent years, its anti-inflammatory effects have been deeply studied and have indicated that HCT is clinically applicable in the treatment of inflammatory diseases. The anti-inflammatory mechanism of HCT is closely associated with inflammatory cells, particularly those that secrete cytokines. The antiviral, antibacterial and immunostimulant effects of HCT are also being elucidated.

\section{Antiviral activity}

Hand, foot, and mouth disease (HFMD). HFMD is a common viral illness that typically infects infants and children under the age of 5 years. Enterovirus 71 (EV71) and coxsackievirus A16 (CVA16) are the two main pathogens of HFMD, which accounted for $>70 \%$ of the total cases during an outbreak in China in 2008 (6). HCT demonstrated therapeutic efficacy and relative safety when used to treat HFMD during recent epidemics $(7,8)$. However, there have been no microbiological studies validating the usefulness of HCT for treating HFMD.

Chen et al (9) studied the antiviral activity of HCT in a series of experiments in which African green monkey kidney epithelial (Vero) cells were infected with EV71 or CVA16 and treated with various concentrations of an HCT extract for $72 \mathrm{~h}$. The results showed that HCT significantly blocked the cytopathic effect associated with EV71 but did not exhibit significant activity against CVA16 infection. However, an earlier study (10) revealed that HCT was active against CVA16. Therefore, HCT appears to be of high potential value in the treatment of HFMD. 
Severe acute respiratory syndrome (SARS). SARS is a life-threatening type of pneumonia caused by the SARS coronavirus. From late 2002 to mid 2003, the SARS virus infected $>8,000$ people worldwide. An aqueous extract of HCT demonstrated a significant stimulatory effect on the proliferation of mouse splenic lymphocytes, and this effect was found to be dose-dependent (11). HCT also showed an inhibitory effect on symptoms such as fever, sore throat and cough presented in the early stage of SARS (12). Lau et al (11) used flow cytometry to reveal that HCT increased the proportion of $\mathrm{CD}^{+}$ and $\mathrm{CD}^{+} \mathrm{T}$ cells in mouse splenic lymphocytes. Moreover, it significantly increased the secretion of interleukin (IL)-2 and IL-10. In order to investigate the anti-inflammatory activity of HCT that is purported to underlie its anti-SARS effects, Lu et al (1) examined the effects of HCT injection (HCI) in the rat carrageenan-induced pleurisy model and the mouse xylene-induced ear edema model. In the pleurisy model, the injection of carrageenan into the pleural cavity resulted in protein-rich fluid accumulation and leukocyte infiltration in the pleural cavity, with the peak inflammatory response occurring after $24 \mathrm{~h}$, when the fluid volume, protein concentration, $\mathrm{C}$-reactive protein and cell infiltration were maximal. These parameters were attenuated by $\mathrm{HCI}$ at various doses, with the strongest inhibitory effect occurring at a dose of $0.54 \mathrm{ml} / 100 \mathrm{~g}$. HCI exhibited similar effects in the mice ear edema model, with $50 \%$ inhibition at a dose of $80 \mu \mathrm{l} / 20 \mathrm{~g}$. The results clearly indicate that HCI has anti-inflammatory activity against the SARS coronavirus.

Herpes simplex virus (HSV) infection. A number of studies (13-15) have demonstrated that $\mathrm{NF}-\kappa \mathrm{B}$ activation plays a significant role in the replication of $\mathrm{HSV}-1$ and HSV-2. HSV infection is efficiently suppressed by the inhibition of $\mathrm{NF}-\kappa \mathrm{B}$ activation. Chen et al (16) found that an aqueous extract of HCT (HCWE) had anti-herpetic activity against HSV-2 and HSV-1 infection, and that this activity was associated with an inhibitory effect on NF- $\mathrm{KB}$ pathway activation.

Pseudorabies herpesvirus (PrV). PrV is a member of the Alphaherpesvirinae subfamily. Piglets infected with PrV die within a few days (17). Ren et al (17) used Vero cells and swine testis cells to investigate the effect of HCI on cell infection by PrV. They found that HCI efficiently inhibited cell infection following the incubation of $\operatorname{PrV}$ with the drug.

Infectious bronchitis virus (IBV). Avian IBV is a type of coronavirus that causes infectious bronchitis. In a study conducted in chicken embryos and chickens (18), it was found that HCT inhibited IBV infection by $>90 \%$ in chicken embryo kidney cells, and reduced IBV-induced apoptosis by $>90 \%$ in vivo and in vitro. HCT fully protected the embryos against IBV challenge and had $>50 \%$ protective effect in chickens in the study.

Dengue virus. Dengue virus is a virus that causes dengue fever, dengue hemorrhagic fever and dengue shock syndrome. A study conducted by Leardkamolkarn et al (19) provided scientific data to support the phytomedicinal properties of HCWE as an inhibitor of the dengue virus. The study identified that hyperoside is the major bioactive compound that effectively acts against dengue infection. The HCT extract was shown to be non-toxic and may be worthy of further development.

\section{Antibacterial activity}

Staphylococcus aureus. Houttuynin is a keto-substituted aldehyde that can be isolated from HCT. Liu et al (20) found that sodium houttuyfonate ( $\mathrm{SH}$ ), an addition compound of sodium bisulfite and houttuynin, exhibited antibacterial activity against 21 strains of Staphyloccus aureus grown in planktonic culture in vitro. Their results showed that the levels of autolysin atl, slel, cidA and lytN transcripts in the SH-treated strain were decreased compared with those in the control strain, and SH diminished the amounts of extracellular DNA of the 21 strains of Staphyloccus aureus in a dose-dependent manner. Furthermore, Lu et al (21) found that SH demonstrated in vitro antibacterial activity against methicillin-resistant Staphylococcus aureus isolates.

\section{Antiparasitic activity}

The leaves of HCT are used by the native Naga tribes of northeast India as a traditional cure for intestinal helminthic infections (22). The anticestodal efficacy of HCT leaf extract has been investigated in vivo against Hymenolepis diminuta in experimentally infected albino rats. Yadav AK and Temjenmongla (22) compared the effect of HCT leaf extract with that of the reference drug praziquantel, and concluded that the HCT leaf extract had anticestodal efficacy.

\section{Other anti-inflammatory activity}

Membranous glomerulonephritis. Pan et al (23) investigated the effect of $\mathrm{SH}$ on the membranous glomerulonephritis (MGN) induced by cationic bovine serum albumin (C-BSA) in BALB/c mice. The C-BSA-induced increases in urinary protein and changes in morphology were attenuated by treatment with SH. The expression levels of nuclear $\mathrm{NF}-\kappa \mathrm{B}$ and monocyte chemotactic protein 1 (MCP-1) were inhibited by $\mathrm{SH}$ in a dose-dependent manner. This study indicated that $\mathrm{SH}$ acted against $\mathrm{C}$-BSA-induced MGN in the mice by suppressing the activation on $\mathrm{NF}-\kappa \mathrm{B}$ and the expression of MCP-1.

Li et al (24) conducted a study to investigate the effect of HCWE on mast cell-mediated anaphylactic reactions. It was observed that the oral administration of HCWE inhibited systemic anaphylaxis in mice as well as the local allergic reaction activated by anti-dinitrophenyl $\operatorname{IgE}$ antibody in rats. The authors also found that HCWE dose-dependently inhibited histamine release and calcium uptake by rat peritoneal mast cells. HCWE increased the intracellular level of cyclic adenosine monophosphate (cAMP) and markedly reduced mast cell activator-induced cAMP expression. These results indicate that HCWE may be useful for the prevention of mast cell-mediated anaphylactic response.

Nonsteroidal anti-inflammatory drugs (NSAIDs) have been shown to be significantly efficacious in the treatment of various inflammatory disorders. NSAIDs function by binding to cyclooxygenase enzymes to inhibit the production of prostaglandins from the substrate arachidonic acid. A study showed 
that Houttuynia cordata supercritical extract (HSE) was able to reduce the production of tumor-necrosis factor (TNF)- $\alpha$ and prostaglandin E2 (PGE2) in a carrageenan-air pouch model of inflammation in mice, which indicates that HSE exerts anti-inflammatory effects by inhibiting TNF- $\alpha$ and cyclooxygenase II-PGE2 pathways $(25,26)$. Therefore, HSE is a potential drug candidate for the relief of various types of inflammation that are responsive to NSAIDs. Thus, it may be useful for reducing the symptoms associated with rheumatoid arthritis (RA), without the marked gastrointestinal side-effects of NSAIDs. Studies have also indicated that HCT is able to efficiently inhibit IL-6, IL-8 and TNF- $\alpha$ production $(27,28)$.

\section{Effects on immunity}

The continuous ingestion of a probiotic fermented four-herb combination (PFH) including HCT has been found to markedly increase lysozymal activity in the serum and spleen, in addition to increasing peripheral blood mononuclear cell $(\mathrm{PBMC})$ proliferation, the $\mathrm{CD} 4^{+}: \mathrm{CD}^{+} \mathrm{T}-1 y m p h o c y t e$ ratio in the spleen, and antibody production levels in broiler chicks (29). Conversely, PGE2 synthesis in serum and PBMC culture medium was significantly decreased in the PFH-fed chicks in comparison with that in the control group in a dose-dependent manner. When the chicks were experimentally infected with Salmonella gallinarum, feeding with $2 \% \mathrm{PFH}$ delayed mortality. Furthermore, the survival rate in the $2 \%$ $\mathrm{PFH}$-fed group was higher than that in the $1 \%$ PFH-fed group and the control group throughout the experimental period. These findings suggest that PFH enhances immune activity in broiler chicks and increases the survival of experimentally infected broiler chicks, possibly by stimulating nonspecific immune responses. Therefore, probiotics and herbs including HCT may provide an alternative to antibiotics and could avoid the development of antibiotic-resistant bacteria by promoting immune activity and preventing disease. Moreover, Lee et al found HCT showed an efficient immunomodulation effect (30). In one study, the usefulness of HCT fractions as an ethnopharmacological treatment for Th2-mediated inflammation was reported to occur through the downregulation of the production of Th2 cytokines (31).

RA is the most common inflammatory arthritis. It is of unknown etiology and affects $0.5-1 \%$ of the general population worldwide $(32,33)$. The condition features repeated outbreaks with an acute or chronic course. Considering the far-ranging aspects for the usage of HCT, it has the potential to be used as a new medicine for the treatment of RA in the near future.

\section{Conclusion}

Existing studies and prior knowledge demonstrate that HCT has antiviral, antibacterial and immunostimulant activities, as well as anti-inflammatory functions. However, whether there are any toxic side-effects or untoward reactions remains unclear. Adverse effects of lead-based traditional preparation techniques may potentially arise if there are any deficiencies in the preparation methods. Therefore, these issues require further evaluation to validate the safety of HCT and support the broadening of its clinical use in the future.
The primary inflammatory site of RA is synovial proliferation and it is the trigger or progression of joint destruction in patients with RA. Thus, the inhibition of synovial hyperplasia in the early course of RA may play a potential therapeutic approach for the treatment of patients with the condition. Moreover, since HCT possesses a broad range of anti-inflammatory functions, such as antiviral, antibacterial and its immunostimulant effect, it may be useful in the treamtent of acute or chronic inflammation as part of RA. However, further studies are required in order to improve the understanding of the role of HCT in RA.

\section{References}

1. Lu HM, Liang YZ, Yi LZ and Wu XJ: Anti-inflammatory effect of Houttuynia cordata injection. J Ethnopharmacol 104: 245-249, 2006.

2. Kang C, Lee H, Hah DY, et al: Protective effects of Houttuynia cordata Thunb. on gentamicin-induced oxidative stress and nephrotoxicity in rats. Toxicol Res 29: 61-67, 2013.

3. Miyata M, Koyama T and Yazawa K: Water extract of Houttuynia cordata Thunb. leaves exert anti-obesity effects by inhibiting fatty acid and glycerol absorption. J Nutr Sci Vitaminol (Tokyo) 56: 150-156, 2010.

4. Hayashi K, Kamiya M and Hayashi T: Virucial effects of the steam distillate from Houttuynia cordata and its components on HSV-1, influenza virus and HIV. Planta Med 61: 237-241, 1995.

5. Chang JS, Chiang LC, Chen CC, Liu LT, Wang KC and Lin CC: Antileukemic activity of Bidens pilosa L. var. minor (Blume) Sherff and Houttuynia cordata Thunb. Am J Chin Med 29: 303-312, 2001

6. Yang F, Ren L, Xiong Z, et al: Enterovirus 71 outbreak in the People's Republic of China in 2008. J Clin Microbiol 47: 2351-2352, 2009

7. Xue BL, Yao ZH and Yu RM: Studies on anti-EV71 virus activity of traditional Chinese medicine and its clinical application in treatment of HFMD. Zhongguo Zhong Yao Za Zhi 36: 3366-3370, 2011 (In Chinese).

8. Cao HJ, Liu ZL, Steinmann P, Mu YJ, Luo H and Liu JP: Chinese herbal medicines for treatment of hand, foot and mouth disease: A systematic review of randomized clinical trials. Eur J Integr Med 4: e85-e111, 2012.

9. Chen XQ, Wang CY, Xu LF, et al: A laboratory evaluation of medicinal herbs used in China for the treatment of hand, foot and mouth disease. Evid Based Complement Alternat Med 2013: 504563, 2013

10. Lin TY, Liu YC, Jheng JR, et al: Anti-enterovirus 71 activity screening of Chinese herbs with anti-infection and inflammation activities. Am J Chin Med 37: 143-158, 2009.

11. Lau KM, Lee KM, Koon CM, et al: Immunomodulatory and anti-SARS activities of Houttuynia cordata. J Ethnopharmacol 118: 79-85, 2008.

12. Li S, Wang RQ, Zhang YL, et al: Symptom combinations associated with outcome and therapeutic effects in a cohort of cases with SARS. Am J Chin Med 34: 937-947, 2006.

13. Patel A, Hanson J, McLean TI, et al: Herpes simplex type 1 induction of persistent NF-kappaB nuclear translocation increases the efficiency of virus replication. Virology 247: 212-222, 1998.

14. Gregory D, Hargett D, Holmes D, Money E and Bachenheimer SL: Efficient replication by herpes simplex virus type 1 involves activation of the IkappaB kinase-IkappaB-p65 pathway. J Virol. 78: 13582-13590, 2004.

15. Yedowitz JC and Blaho JA: Herpes simplex virus 2 modulates apoptosis and stimulates NF-kappaB nuclear translocation during infection in human epithelial HEp-2 cells. Virology 342: 297-310, 2005.

16. Chen XQ, Wang ZX, Yang ZY, et al: Houttuynia cordata blocks HSV infection through inhibition of NF- $\mathrm{KB}$ activation. Antiviral Res 92, 341-345, 2011

17. Ren X, Sui X and Yin J: The effect of Houttuynia cordata injection on pseudorabies herpesvirus (PrV) infection in vitro. Pharm Biol 49: 161-166, 2011.

18. Yin J, Lia G, Lia J, Yang Q and Ren X: In vitro and in vivo effects of Houttuynia cordata on infectious bronchitis virus. Avian Pathol 40: 491-498, 2011. 
19. Leardkamolkarn V, Sirgulpanit W, Phurimsak C, Kumkate S, Himakoun L and Sripanidkulchai B: The inhibitory actions of Houttuynia cordata aqueous extract on dengue virus and dengue-infected cells. J Food Biochem 36: 86-92, 2010.

20. Liu G, Xiang H, Tang X, et al: Transcriptional and functional analysis shows sodium houttuyfonate-mediated inhibition of autolysis in Staphylococcus aureus. Molecules 16: 8848-8865, 2011

21. Lu X, Yang X, Li X, et al: In vitro activity of sodium new houttuyfonate alone and in combination with oxacillin or netilmicin against methicillin-resistant Staphylococcus aureus. PLoS One 8: e68053, 2013.

22. Yadav AK and Temjenmongla: Anticestodal activity of Houttuynia cordata leaf extract against Hymenolepis diminuta in experimentally infected rats. J Parasit Dis 35: 190-194, 2011.

23. Pan P, Wang YJ, Han L, Liu X, Zhao M and Yuan YF: Effects of sodium houttuyfonate on expression of NF-kappaB and MCP-1 in membranous glomerulonephritis. J Ethnopharmacol 131: 203-209, 2010

24. Li GZ, Chai OH, Lee MS, Han EH, Kim HT and Song CH: Inhibitory effects of Houttuynia cordata water extracts on anaphylactic reaction and mast cell activation. Biol Pharm Bull 28: 1864-1868, 2005.

25. Li W, Zhou P, Zhang Y and He L: Houttuynia cordata, a novel and selective COX-2 inhibitor with anti-inflammatory activity. J Ethnopharmacol 133: 922-927, 2011.

26. Shin S, Joo SS, Jeon JH, et al: Anti-inflammatory effects of a Houttuynia cordata supercritical extract. J Vet Sci 11:273-275, 2010.
27. Park E, Kum S, Wang C, Park SY, Kim BS and Schuller-Levis G: Anti-inflammatory activity of herbal medicines: inhibition of nitric oxide production and tumor necrosis factor-alpha secretion in an activated macrophage-like cell line. Am J Chin Med 33: 415-424, 2005.

28. Lee HJ, Seo HS, Kim GJ, et al: Houttuynia cordata Thunb inhibits the production of pro-inflammatory cytokines through inhibition of the NFKB signaling pathway in HMC-1 human mast cells. Mol Med Rep 8: 731-736, 2013.

29. Jung BG, KO JH and Lee BJ: Dietary supplementation with a probiotic fermented four-herb combination enhances immune activity in broiler chicks and increases survivability against Salmonella gallinarum in experimentally infected broiler chicks. J Vet Med Sci 72: 1565-1573, 2010.

30. Lee JS, Kim IS, Kim JH, Kim JS, Kim DH and Yun CY: Suppressive effects of houttuynia cordata Thunb (Saururaceae) extract on Th2 immune response. J Ethnopharmacol 117: 34-40, 2008.

31. Gabriel SE and Michaud K: Epidemiological studies in incidence, prevalence, mortality and comorbidity of the rheumatic diseases. Arthritis Res Ther 11: 229, 2009.

32. Yilmaz S and Simek I: Early intervention in the treatment of rheumatoid arthritis: focus on tocilizumab. Ther Clin Risk Manag 9: 403-408, 2013.

33. Athanasiadou S, Githiori J and Kyriazakis I: Medicinal plants for helminth parasite control: facts and fiction. Animal 1: 1392-1400, 2007. 